\title{
Removal of Traumatic Hematic Cyst of the Orbit Through Sub-Brow Approach
}

\author{
Doyeon Kim, MD, Jang Wook Gwak, MD, Sung Seok Ryu, MD, and Myeong Sang Yu, MD, PhD \\ Department of Otorhinolaryngology-Head and Neck Surgery, Asan Medical Center, University of Ulsan College of Medicine, \\ Seoul, Republic of Korea
}

Orbital hematic cyst is characterized by cystic accumulation of hematogenous debris surrounded by a wall of fibrous tissue without an epithelial lining. It is a relatively uncommon benign cyst and occurs secondary to blunt trauma in most cases. We experienced a case of 53-year-old male who presented with proptosis of the left eye. A cystic lesion was found at the superolateral orbital rim of the patient. We report a case of orbital hematic cyst that was resected completely through the sub-brow approach.

Keywords: Hematic cyst; Incision below eyebrow; Orbit.

\section{INTRODUCTION}

Intraorbital hemorrhage is a rare condition known to be mostly caused by blunt trauma [1]. If the hematoma is not absorbed naturally, it can progress to a space-occupying mass called a subperiosteal hematic cyst. Hematic cyst in the orbit is a rare condition distinguished from cavernous malformation, lymphatic malformation, and dermoid cyst by a lack of endothelium [2]. Hematic cysts are reported to develop across a wide timeframe, from 10 days after the trauma to up to 20 years after the trauma, and may be divided into acute and chronic hematic cyst [3]. Acute hematic cyst lack the thick capsule and cholesterol cleft, and shows post-trauma exophthalmos, conjunctival chemosis, and limitations of extraocular movement. In contrast, chronic hematic cyst causes bone erosion, and is made up of cholesterol cleft, hemosiderin, giant cells, and fibrous pseudocapsule [4]. It tends to be discovered months or even years after the trauma. This case reports a successful treatment of traumatic hematic cyst, which had progressed to the intraconal space of the orbit, through an in-

Received: June 22, 2021 Revised: July 20, 2021

Accepted: July 26, 2021

Address for correspondence: Myeong Sang Yu, MD, PhD, Department of Otorhinolaryngology-Head and Neck Surgery, Asan Medical Center, University of Ulsan College of Medicine, 88 Olympic-ro 43-gil, Songpa-gu, Seoul 05505, Republic of Korea

Tel: +82-2-3010-3710, Fax: +82-2-489-2773, E-mail: dryums@gmail.com This is an Open Access article distributed under the terms of the Creative Commons Attribution Non-Commercial License (https://creativecommons.org/licenses/bync/4.0) which permits unrestricted non-commercial use, distribution, and reproduction in any medium, provided the original work is properly cited. cision made below the eyebrow, as well as the literature review pertaining to the case.

\section{CASE REPORT}

A 53-year-old male patient was admitted to the hospital with symptoms of exophthalmos in his left eye, which had progressed gradually from 5 years before the admission (Fig. 1). The patient had a past medical history of blunt trauma to the left forehead and the temporal region, and had no past history of nasal surgeries. The outpatient examination undertaken at the ophthalmology department of the hospital confirmed an exophthalmos of $4 \mathrm{~mm}$ in the left eye, but both eyes showed a normal visual acuity of 1.0 and eye pressure of $20 \mathrm{~mm} \mathrm{Hg}$ in both eyes, and had no unusual presentation in the funduscopic and visual field examination. A mass with a size of $2.2 \times 2.8 \mathrm{~cm}$ was identified with no contrast-enhancement in the superior temporal quadrant of the frontozygomatic suture, the most common location of dermoid cyst, in the computerized tomography (CT) undertaken before the surgery. Dural invasion was not identified in the T2-weighted magnetic resonance imaging (MRI). High signal intensity was observed at the center of the mass, and heterogeneous signal intensity to the outer areas of the mass. Contrast enhancement was found to be homogenous in T1-weighted contrast-enhancement MRI (Fig. 2). Integration of the CT and MRI images allowed for the consideration of dermoid cyst through differential diagnosis. Manifestation of a cyst close to the frontal sinus also led to the consideration of mucocele from the 
obstruction of the sinus through differential diagnosis as well $[5,6]$. The surgery was conducted through an incision made below the eyebrow to minimize scarring after surgery (Fig. 3). A $3 \mathrm{~cm}$ incision was made along the lower rim of the eyebrow, followed by an incision to the orbicularis oculi muscle, through an upper approach made from the outer side of the orbicularis oculi. This allowed the approach from the area immedi- ately below the upper orbital rim via a tear toward the frontal bone, thus accessing the subperiosteal space. A cyst containing a brown fluid was identified just above the periorbita. In order to ensure the complete removal of the cyst wall, curets and drills were used to remove as much of the cyst wall as possible from the bone and the periorbita. There were no diplopia, limitation of extraocular movement, and other compli-
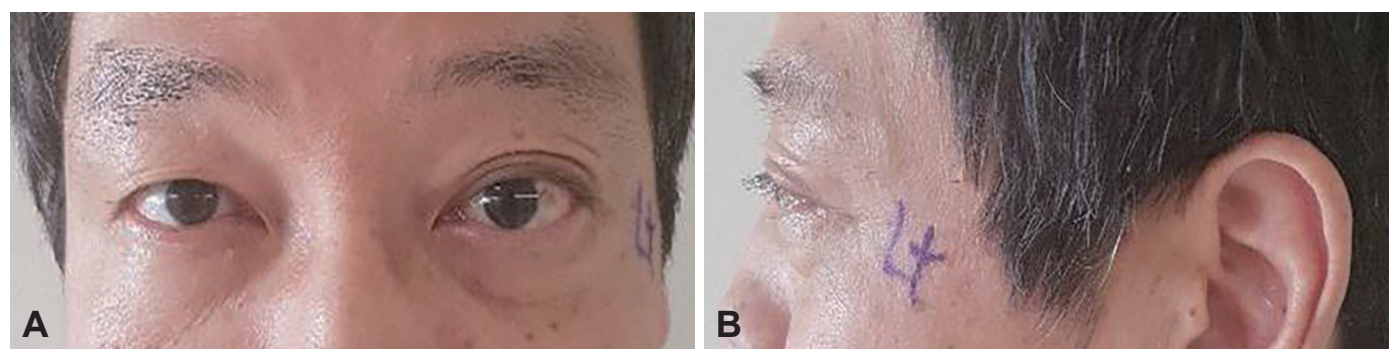

Fig. 1. Preoperative facial photos. A: Frontal view of preoperative facial photo. B: Lateral view of preoperative facial photo.
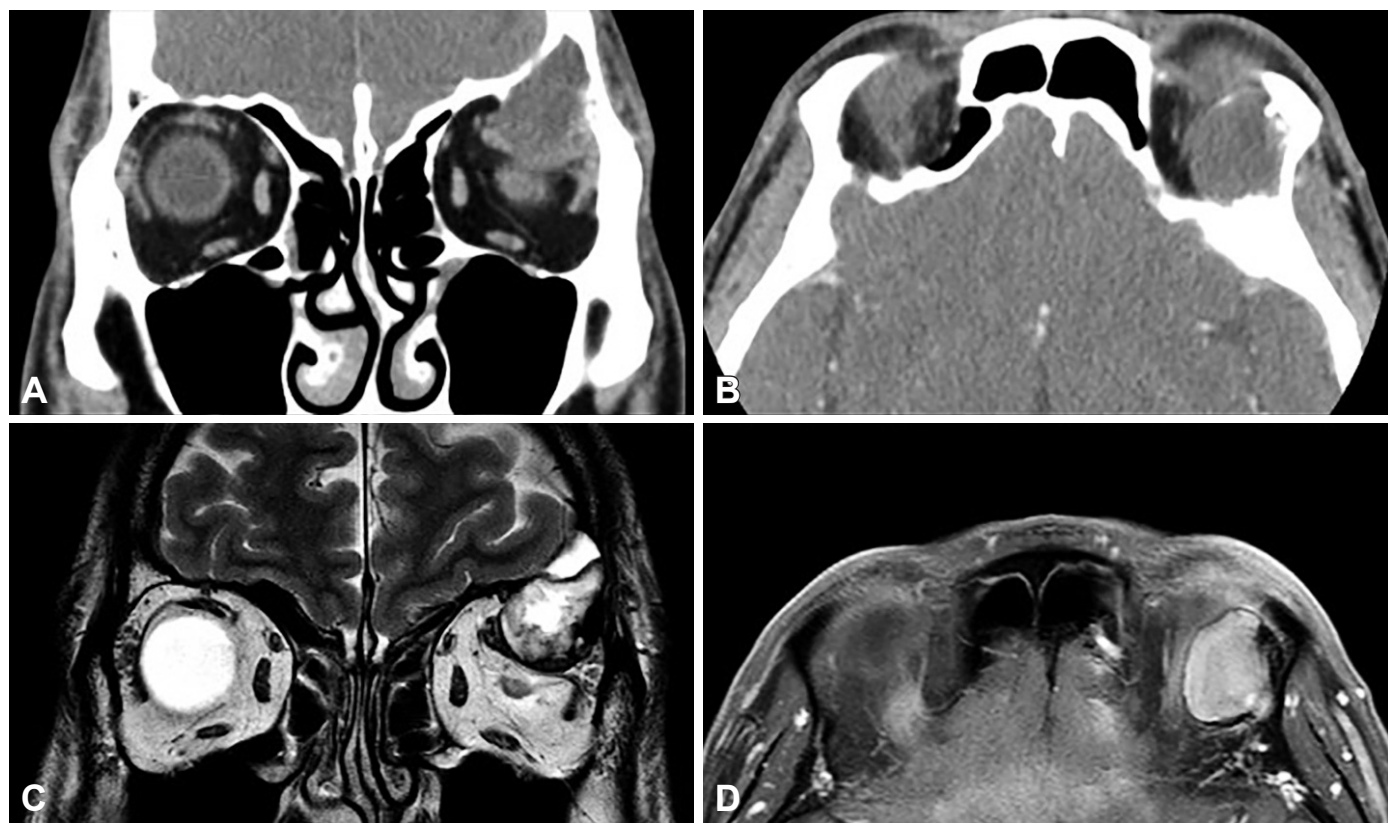

Fig. 2. Preoperative radiological imaging. A: Coronal view of contrast computed tomography (CT) scan represents about $2 \mathrm{~cm}$-sized cyst was pressing down the superior rectus muscle inferomedially. B: Axial view of contrast CT shows that non-enhancing cyst was extending to intraconal space of the orbit. C: In T2-weighted magnetic resonance imaging (MRI) scan, a cyst which originated from superolateral orbital rim showed internal high signal density. D: In contrast-enhanced T1-weighted MRI scan, well enhancing lesion was identified.
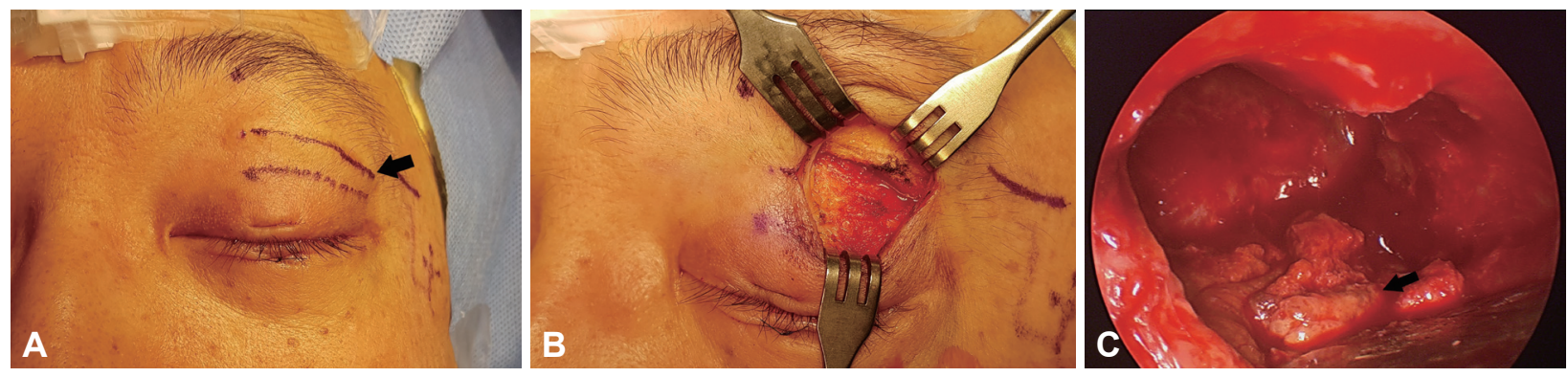

Fig. 3. Intraoperative findings. A: Sub-brow incision line was drawn (arrow). B: Upper portion of the orbital rim was exposed by sub-brow incision. C: Yellowish non-epithelial contents of the orbital hematic cyst was identified (arrow). 


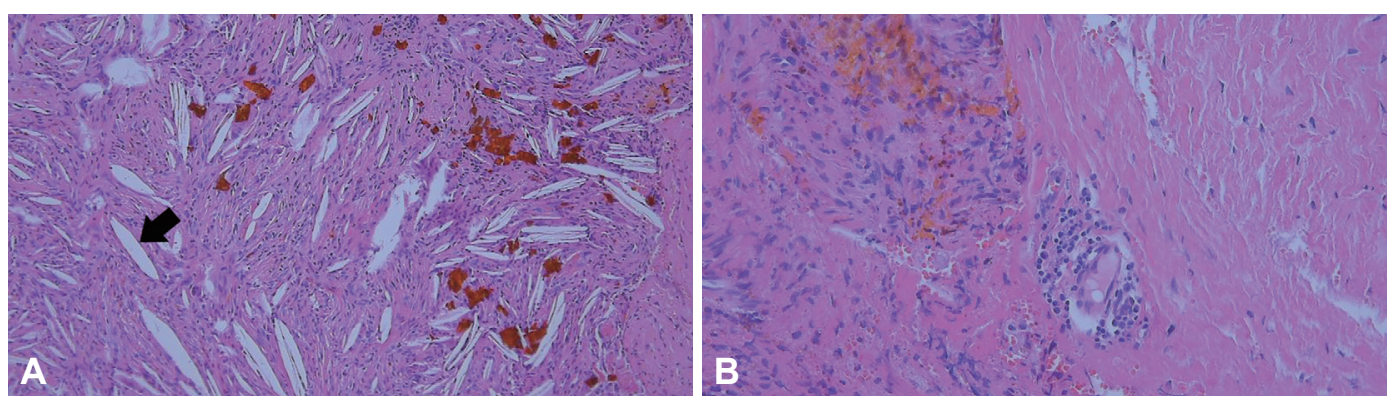

Fig. 4. Pathologic findings. A: Cholesterol clefts (arrow) due to lipid-laden macrophages. Yellow-brownish crystalloid materials suggesting old hemorrhage (Original magnification $\times 100$, hematoxylin and eosin). B: Collections of red blood cells without vascular lining surrounded by formation of fibrous tissues (Original magnification $\times 300$, hematoxylin and eosin).

cations after the surgery [7]. The surgery successfully resolved the exophthalmos of the left eye. A pathology diagnosis using hematoxylin and eosin stain reported the finding of cholesterol cleft, which is manifested by the absorption of old hematoma to the macrophage, and yellow-brown crystalline materials surrounded by fibrous capsule with a lack of epithelial wall (Fig. 4). Based on this finding, a hematic cyst was diagnosed [2]. Two years of outpatient follow-up examination revealed no complications or recurrence.

\section{DISCUSSION}

Differential diagnosis for orbital cyst conditions may consider hematic cyst, mucocele, and dermoid cyst [8]. This case originally assessed the possibility of a hematic cyst as low prior to the surgery, but was able to diagnose the hematic cyst through post-surgery pathology diagnosis. Symptoms of hematic cyst include exophthalmos, limitations of extraocular movement, hydroblepharon, and reduced visual acuity. The patient's past medical history of trauma or sinus surgery must be consulted in detail to identify the hematic cyst. Most patients suffering from hematic cyst have a past medical history of trauma [9]. However, spontaneous hemorrhage can also cause hematic cyst independently from trauma [2]. Known causes of non-traumatic hemorrhage include vascular lesions, increased venous pressure in the cranial vein, hemorrhagic disorders, infections, and tumor [10]. Furthermore, the association between the orbital floor fracture repair and hematic cyst is well-documented. The usage of alloplastic implants in the repair of orbital floor fracture, and the subsequent erosion of the surrounding periosteum, is thought to be the cause of such hematic cyst [11]. Radiographical investigation is a core part of the diagnosis for hematic cyst [8]. If hematic cyst is strongly suspected, there is no need for the cyst wall to be completely removed; this is the reason why a diagnostic imaging using CT or MRI are important in preparing for a surgery to address the hematic cyst. In CT, hematic cyst appears as a homogeneous ulcer with a clear boundary located below the periosteum without contrast-enhancement. MRI can be used to confirm the existence of blood within the cyst, which can be distinguished into five stages based on the timeframe of the hemorrhage. Hyperacute period shows isointensity in both T1 and T2-weighted images. In the acute period, one or two days after the hemorrhage, signal intensity in the T2-weighted image goes down. In the early subacute period (2 to 7 days), signal intensity in T1-weighted image goes up, while the later subacute period (7 to 28 days) has the signal intensity of the T2-weighted images increase. Once in the chronic period ( 28 days and onward), the surrounding areas show low signal intensity in both $\mathrm{T} 1$ and $\mathrm{T} 2$-weighted images, while the center shows isointensity in T1-weighted image, and high signal intensity in T2-weighted image [10]. Pathologically speaking, hematic cyst is made up of fibrous granulation tissues and the blood degradation products. The cyst wall is made up of a dense connective tissue. Hemosiderin within the cyst shows that the cyst has been growing chronically. The size of the hematic cyst is known to increase from repeated hemorrhage, hemostasis, and fibrinolysis [12]. Needle aspiration of the hematic cyst can achieve improvements in most of the eye and orbital symptoms [3]. However, needle aspiration bears a risk of recurrence. Surgical removal of the cyst is recommended as a treatment [13]. Furthermore, venous imaging or pathology diagnosis reveal that the great majority of the patients suffering from non-traumatic hemorrhage also have venous malformations as well, which can increase the risk of needle aspiration over that of surgical removal [14]. During surgery, care should be taken to prevent the damages to the tissue surrounding the eye, extraocular muscle, and the orbit. Surgical treatment of the cyst at the frontozygomatic suture can utilize a variety of approaches. The first option, direct incision over the cyst, can result in scarring after the surgery. Incision on the eyebrow can result in cosmetic problems after the surgery, as the eyebrows fail to grow back after the surgery. Approach from the upper eyelid has the advantage of the scar being invisible and requiring simple surgical skills, and thus can be a good choice. A report on the treatment of dermoid cyst in the 
frontozygomatic suture region involving 34 children has reported the successful utilization of the upper eyelid approach [15]. Depending on the location of the cyst, such as in this case, an incision can also be made below the eyebrow. Incision below the eyebrow also has the advantage of invisible scarring and adequate operative fields. As this case suggests, the diagnosis of hematic cyst may be considered for patients suffering from exophthalmos who have a past medical history of head trauma. In that case, depending on the location of the cyst, an incision below the eyebrow can ensure adequate operative field for the surgical removal of the cyst.

\section{Ethics Statement}

Written and verbal consent for publication was obtained from the patient.

\section{Availability of Data and Material}

The datasets generated or analyzed during the study are available from the corresponding author on reasonable request.

\section{Conflicts of Interest}

The authors have no potential conflicts of interest to disclose.

\section{Author Contributions}

Conceptualization: Doyeon Kim, Myeong Sang Yu. Data curation: all authors. Investigation: all authors. Writing-original draft: Doyeon Kim, Myeong Sang Yu. Writing_reiew \& editing: Doyeon Kim, Myeong Sang Yu.

\section{ORCID iDs}

Doyeon Kim Jang Wook Gwak Sung Seok Ryu https://orcid.org/0000-0003-2611-616X Myeong Sang Yu https://orcid.org/0000-0001-5200-0609 https://orcid.org/0000-0001-5861-7386

Funding Statement None

\section{REFERENCES}

1) Kim UR, Arora V, Shah AD, Solanki U. Clinical features and management of posttraumatic subperiosteal hematoma of the orbit. Indian J Ophthalmol 2011;59(1):55-8.

2) Yoshikawa K, Fujisawa $H$, Kajiwara K, Fujii M, Kato S, Akimura T, et al. Cause of hematic cysts of the orbit: increased fibrinolysis and immunohistologic expression of tissue plasminogen activator. Ophthalmology 2009;116(1):130-4

3) Seigel RS, Williams AG, Hutchison JW, Wolter JR, Carlow TJ, Rogers DE. Subperiosteal hematomas of the orbit: angiographic and computed tomographic diagnosis. Radiology 1982;143(3):711-4.

4) Friedberg MH, David O, Woog J, Heilman CB. Orbital hematic cyst: case report and clarification of terms. Skull Base Surg 1997;7(2):95-9.

5) Bartlett SP, Lin KY, Grossman R, Katowitz J. The surgical management of orbitofacial dermoids in the pediatric patient. Plast Reconstr Surg 1993;91(7):1208-15.

6) Devars du Mayne M, Moya-Plana A, Malinvaud D, Laccourreye O, Bonfils P. Sinus mucocele: natural history and long-term recurrence rate. Eur Ann Otorhinolaryngol Head Neck Dis 2012;129(3):125-30.

7) Pryor SG, Lewis JE, Weaver AL, Orvidas LJ. Pediatric dermoid cysts of the head and neck. Otolaryngol Head Neck Surg 2005;132(6):938-42.

8) Kaufman LM, Villablanca JP, Mafee MF. Diagnostic imaging of cystic lesions in the child's orbit. Radiol Clin North Am 1998;36(6):1149-63.

9) Yazici B, Gönen T. Posttraumatic subperiosteal hematomas of the orbit in children. Ophthalmic Plast Reconstr Surg 2011;27(1):33-7.

10) McNab AA. Nontraumatic orbital hemorrhage. Surv Ophthalmol 2014;59(2):166-84

11) Glavas I, Lissauer B, Hornblass A. Chronic subperiosteal hematic cyst formation twelve years after orbital fracture repair with alloplastic orbital floor implant. Orbit 2005;24(1):47-9.

12) Cameron JD, Letson RD, Summers CG. Clinical significance of hematic cyst of the orbit. Ophthalmic Plast Reconstr Surg 1988;4(2):95-9.

13) Iwata A, Matsumoto T, Mase M, Yamada K. Chronic, traumatic intraconal hematic cyst of the orbit removed through the fronto-orbital approach--case report. Neurol Med Chir (Tokyo) 2000;40(2):106-9.

14) Krohel GB, Wright JE. Orbital hemorrhage. Am J Ophthalmol 1979; 88(2):254-8.

15) Nelson KE, Mishra A, Duncan C. Upper blepharoplasty approach to frontozygomatic dermoid cysts. J Craniofac Surg 2011;22(6):e41-4. 